\title{
DEFECTIVE VON WILLEBRAND FACTOR AND ANGIOPOIETIN-2 RELEASE FROM VON WILLEBRAND DISEASE PATIENTS' BLOOD OUTGROWTH ENDOTHELIAL CELLS
}

\author{
R Starke, ${ }^{1}$ K Paschalaki, ${ }^{1}$ C Dyer, ${ }^{2}$ K Harrison-Lavoie, ${ }^{2} \mathrm{~J}$ Cutler, ${ }^{3} \mathrm{~T}$ McKinnon, ${ }^{4}$ \\ C Millar, ${ }^{4}$ D Cutler, ${ }^{2}$ M Laffan, ${ }^{4}$ A Randi ${ }^{5}{ }^{1}$ Vascular Science, NHLI, Imperial College \\ London; ${ }^{2}$ MRC-LMCB, University College London; ${ }^{3}$ Molecular Haemostasis Laboratory, \\ GSTS Pathology; ${ }^{4}$ Haematology Department, Imperial College London; ${ }^{5}$ Vascular \\ Science, NHLI, Imperial College London
}

doi:10.1136/heartjnl-2013-304019.189

Von Willebrand factor (VWF) mediates platelet adhesion to damaged endothelial cells (EC) and stabilises blood coagulation factor VIII. In EC, VWF is stored within and directs the formation

of Weibel-Palade bodies (WPB), which contain many proteins including the angiogenic regulator Angiopoietin (Ang)-2. Von Willebrand disease (VWD), the most common inherited bleeding disorder is caused by decrease (type 1 and 3) or dysfunction (type 2) of VWF. VWD can be associated with angiodyplasia, describing the presence of vascular lesions which can cause intractable bleeding. We demonstrated a role for VWF in angiogenesis and hypothesized that angiodysplasia might be linked to dysregulated angiogenesis through several pathways. VWD is a heterogenous disorder and a wide range of mutations in the VWF gene have been characterised; however their cellular consequences are still poorly understood. We have used a recently developed approach to study the molecular and cellular basis of VWD and angiogenesis. Mononuclear cells were isolated from peripheral blood of four type 1 VWD, four type 2 VWD patients and nine healthy controls and cultured to obtain blood outgrowth EC (BOEC). We confirmed the endothelial lineage of BOEC by staining for various EC markers. Confocal microscopy confirmed VWF and Ang-2 co-staining within BOEC WPB. We measured VWF mRNA and protein levels, both before and after stimulation, and VWF multimers. Decreased mRNA levels were predictive of plasma VWF levels in type 1 VWD, confirming a defect in VWF synthesis (Pearson $\mathrm{R}=0.9976$, $\left.p=0.0024^{* *}, r^{2}=0.9952\right)$. However BOEC from this group of patients also showed defects in processing, storage and/or secretion of VWF. Two patients with type 1 VWD showed increased levels of pro-peptide containing VWF one of which showed expansion of the endoplasmic reticulum consistent with retention of defective VWFThree type 1 patients showed no Ang-2 release post-cellular stimulation, consistent with the inability of these BOEC to correctly package WPB. One patient showed significant release of Ang-2 $(\mathrm{P}<0.05)$; interestingly this patient showed the highest levels of VWF within the type 1 group with more WPB and VWF strings released upon stimulation. Levels of VWF mRNA and protein were normal in BOEC from three type 2 VWD patients, supporting the dysfunctional VWF model. However, one type $2 \mathrm{M}$ patient showed decreased VWF synthesis and storage, indicating a complex cellular defect. All type 2 patients' BOEC show a significant release of Ang-2 upon stimulation, confirming correct packaging of WPB. Together our data suggest that the angiogenesis defect in type 1 VWD patients is due to dysregulation of Ang-2 storage and release, but that other mechanisms may be more relevant in type 2 VWD. These results demonstrate for the first time that isolation of endothelial cells from VWD patients provides novel insight into the cellular mechanisms of the disease. 